\title{
Does the mesodermal derangement in Chiari Type I malformation extend to the cervical spine? Evidence from an analytical morphometric study on cervical paraspinal muscles
}

\author{
Sumit Thakar, MCh, Avinash Kurudi Siddappa, MS, MCh, Saritha Aryan, MS, MCh, \\ Dilip Mohan, MS, MCh, Narayanam Anantha Sai Kiran, MCh, and Alangar S. Hegde, MCh, PhD
}

Department of Neurological Sciences, Sri Sathya Sai Institute of Higher Medical Sciences, Bangalore, India

\begin{abstract}
OBJECTIVE The mesodermal derangement in Chiari Type I malformation (CMI) has been postulated to encompass the cervical spine. The objectives of this study were to assess the cross-sectional areas (CSAs) of cervical paraspinal muscles (PSMs) in patients with CMI without syringomyelia, compare them with those in non-CMI subjects, and evaluate their correlations with various factors.
\end{abstract}

METHODS In this retrospective study, the CSAs of cervical PSMs in 25 patients were calculated on T2-weighted axial MR images and computed as ratios with respect to the corresponding vertebral body areas. These values and the cervical taper ratios were then compared with those of age- and sex-matched non-CMI subjects and analyzed with respect to demographic data and clinicoradiological factors.

RESULTS Compared with the non-CMI group, the mean CSA values for the rectus capitis minor and all of the subaxial PSMs were lower in the study group, and those of the deep extensors were significantly lower $(p=0.004)$. The cervical taper ratio was found to be significantly higher in the study cohort $(p=0.0003)$. A longer duration of symptoms and a steeper cervical taper ratio were independently associated with lower CSA values for the deep extensors $(p=0.04$ and $p$ $=0.03$, respectively). The presence of neck pain was associated with a lower CSA value for the deep flexors $(p=0.03)$.

CONCLUSIONS Patients with CMI demonstrate alterations in their cervical paraspinal musculature even in the absence of coexistent syringomyelia. Their deep extensor muscles undergo significant atrophic changes that worsen with the duration of their symptoms. This could be related to a significantly steeper cervical taper ratio that their cervical cords are exposed to. Neck pain in these patients is related to atrophy of their deep flexor muscles. A steeper cervical taper ratio and alterations in the PSMs could be additional indicators for surgery in patients with CMI without syringomyelia.

https://thejns.org/doi/abs/10.3171/2016.12.SPINE16914

KEY WORDS cervical paraspinal muscles; morphometry; Chiari Type I malformation

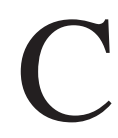

HIARI Type I malformation (CMI) is a mesodermal disorder characterized by an abnormal caudal descent of the cerebellar tonsils through the foramen magnum. The pathophysiological spectrum of the disorder is attributed to altered CSF pressure dynamics in the spinal subarachnoid space and the spinal cord. The ensuing changes in the anterior and/or posterior horn cells have been demonstrated by the occurrence of denervation in the cervical paraspinal muscles (PSMs) in patients with $\mathrm{CMI}$ and syringomyelia. ${ }^{23}$ However, changes in the cer- vical PSMs have not been assessed in patients with CMI in the absence of coexistent syringomyelia. The evaluation of cervical PSMs in CMI gains significance, given that neck pain is a common symptom in this disorder and that alterations in cervical PSM morphology have been reported to be significantly correlated with this symptom. ${ }^{9,14}$ Furthermore, it has also been postulated that the mesodermal derangement in CMI extends to the subaxial cervical spine, $, 8,24$ and it is not known whether these changes involve the cervical PSMs as well.

ABBREVIATIONS CMI = Chiari Type I malformation; CSA = cross-sectional area; CVJ = craniovertebral junction; DE = deep extensor; DF = deep flexor; ICV = intracranial volume; OCA = occipital condyle area; PFV = posterior fossa volume; $\mathrm{PSM}=$ paraspinal muscle; $\mathrm{RCma}=$ rectus capitis major; $\mathrm{RCmi}=$ rectus capitis minor; $\mathrm{SE}=$ superficial extensor; SF = superficial flexor; VBA = vertebral body area; WAD = whiplash-associated disorder.

SUBMITTED August 2, 2016. ACCEPTED December 12, 2016.

INCLUDE WHEN CITING Published online May 12, 2017; DOI: 10.3171/2016.12.SPINE16914. 


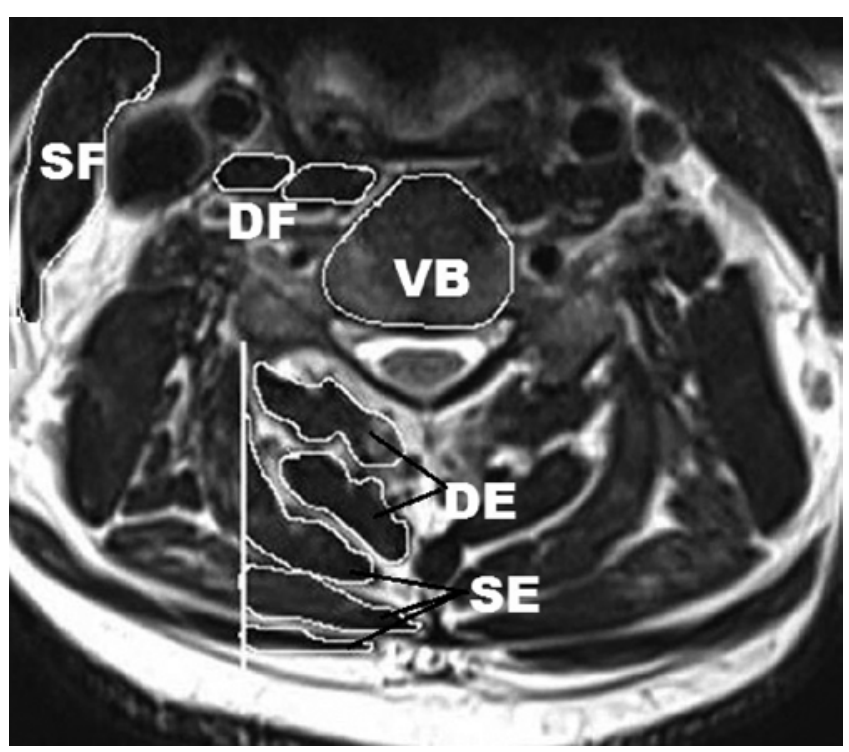

FIG. 1. T2-weighted axial MRI section demonstrating the regions of interest used in measurement of the CSA of the subaxial PSMs. VB = vertebral body.

\section{Methods \\ Patient Population}

Records from a total of 40 symptomatic patients with primary CMI (demonstrating a tonsillar herniation $\geq 5$ $\mathrm{mm}$ below the foramen magnum without any predisposing cause for the same) without syringomyelia, who underwent foramen magnum decompression and duraplasty between 2008 and 2012, were screened. The study cohort was a homogeneous group of 25 patients whose preoperative MR images were stored in the hospital imaging system and who did not have scoliosis, any coexisting bony craniovertebral junction (CVJ) anomaly, any cervical pathology, or any history of surgery in the CVJ or cervical regions. Patients with features of a "presyrinx" condition were excluded from the study.

\section{Comparison Group}

Twenty-five age- and sex-matched non-CMI subjects were selected from the neurosciences outpatient department for comparison of their cervical PSM morphometry and cervical taper ratio with that of the study group. These subjects did not report neck or radicular pain nor previous neck trauma or surgery, and had undergone cervical spine MRI as part of a screening protocol during their neurological evaluation.

\section{Radiographic Evaluation}

The same imaging protocols were used for measurements in both the comparison and study groups. The crosssectional area (CSA) measurement method was based on the technique standardized in previous studies. ${ }^{1,21}$ Digital images of the preoperative cervical spine MRI were retrieved from the hospital radiographic system (Synapse, Fujifilm Health Systems). MR images were acquired on an HDi 1.5-T magnet (GE Signa) using a standard neurovascular coil. Measurement parameters were as follows: slic-
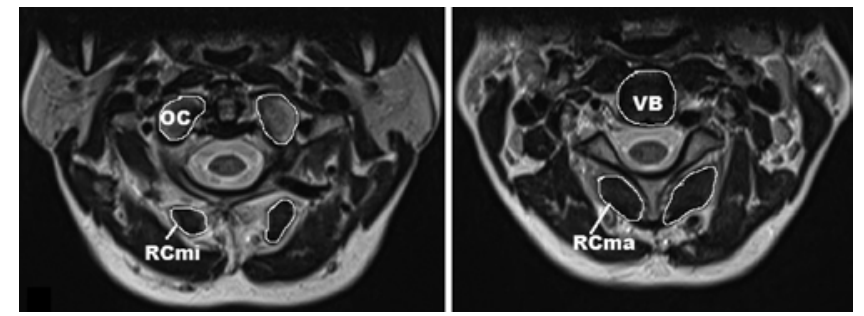

FIG. 2. T2-weighted axial MRI scans demonstrating measurement of the CSA of the RCmi (left) and RCma (right). OC = occipital condyle.

es 26; slice thickness and space $3.7 \mathrm{~mm}$; FOV $180 \times 188$ mm; TR 4360 msec; TE 98.2 msec; matrix size 1.60/256, number of excitations 1.5; and flip angle $90^{\circ}$.

Measurements for the suboccipital muscles (rectus capitis posterior minor [RCmi] and rectus capitis posterior major [RCma]) were taken at occiput- $\mathrm{C} 1$ for the $\mathrm{RCmi}$ muscle after identifying the dens and occipital condyles and at the most cephalad portion of the C-2 vertebral level for the RCma muscle on axial sections of conventional T2weighted spin-echo images. For the measurement of the subaxial PSM areas, axial sections at the upper vertebral endplates from C-3 to C-7 taken parallel to the disc spaces were used. The measured flexors included the sternocleidomastoid (superficial flexor [SF]), and the longus colli and longus capitis (deep flexors [DFs]). The extensors included the multifidus and semispinalis cervicis (deep extensors [DEs]); and the semispinalis capitis, splenius capitis, and upper trapezius (superficial extensors [SEs]) (Fig. 1). The CSA measurement was performed by creating a region of interest for each muscle bilaterally. Perpendicular lines from the lateral border of the facets were used as lateral limits for the region of interest for the SEs.

Muscle CSA/bony CSA ratios rather than absolute muscle CSA values were used to eliminate biases arising from variations in the build of the patient. This was based on a previously used assumption that the muscle areas biomechanically correspond to the morphometry of the bone at any given level. ${ }^{21}$ The occipital condyle areas (OCAs) were used for the calculation of this ratio for the RCmi area values (Fig. 2 left), whereas the vertebral body areas (VBAs) at the cuts taken for measurement of the muscle areas were used for the RCma (Fig. 2 right) and subaxial muscle area values (Fig. 1).

Two consultant neurosurgeons (S.T. and A.K.S.) functioned as independent observers for all measurements. They were blinded to the clinical history, the radiologist's report at the time of imaging, and the group allocation into cases and control subjects. The parasagittal MRI sequences demonstrating the tonsillar position were not provided to them during the CSA measurements. The mean of the sum of the muscle CSAs on either side at all levels, and the means of the PSM CSA/bony CSA ratios were then calculated as follows: RCmi CSA/OCA, RCma CSA/VBA, SF CSA/VBA, DF CSA/VBA, SE CSA/VBA, DE CSA/ VBA, DF CSA/DE CSA, and SF CSA/SE CSA. The mean values of the PSM CSA/bony CSA ratios recorded by the 2 observers were used for analysis.

The measurement of the cervical taper ratio (tapering of the anteroposterior spinal canal dimension from C-1 to 
TABLE 1. Radiological characteristics of the study cohort

\begin{tabular}{lrrr}
\hline \multicolumn{1}{c}{ Variable } & Mean \pm SD & Min & Max \\
\hline PFVIICV ratio & $0.15 \pm 0.02$ & 0.11 & 0.18 \\
\hline Tonsillar herniation $(\mathrm{mm})$ & $13.26 \pm 7.35$ & 5.70 & 29.89 \\
\hline Taper ratio & $0.84 \pm 0.51$ & 0.13 & 2.10 \\
\hline
\end{tabular}

C-7) was done by a previously described technique. ${ }^{8}$ Lines placed perpendicular to the spinal canal at the midpoint between the top and bottom of each vertebra on the T2weighted sagittal MRI sequences were measured as the anteroposterior diameter of the spinal canal and plotted against the spine levels. A trend line was fitted by linear regression to the diameters between $\mathrm{C}-1$ and $\mathrm{C}-7$, and the slope of this line was recorded as the cervical taper ratio $(\mathrm{mm} /$ level). The posterior fossa volume (PFV) and intracranial volume (ICV) were measured as described previously. ${ }^{6}$

\section{Statistical Analysis}

Using the formula for sample size calculation for paired means and incorporating the clinically meaningful difference in CSA values from a previous study on cervical PSMs, ${ }^{21}$ a sample size of 25 was determined to be adequate to obtain a power of 0.9 for the study. Data were entered in an Excel spreadsheet (Microsoft, Inc.) and analyzed using SPSS version 20. Means and standard deviations were computed for continuous variables. The Student t-test was used to compare differences between the area ratios in the comparison and study groups, and for the subgroup analysis based on the absence or presence of neck pain.

Correlation of various continuous variables (age, duration of symptoms, PFV/ICV, tonsillar herniation, and taper ratio) with the muscle area ratios was analyzed using the Pearson's correlation test. An ANOVA was used to analyze the effect of categorical variables (sex, presence or absence of neck pain, and CSF flow on cine MRI) on the muscle area values. A linear regression analysis was performed to predict the muscle area ratios from various clinical and radiological factors. Interobserver variability was measured using the intraclass correlation coefficient and standardized ratings of agreement. ${ }^{11}$

\section{Results}

\section{Demographic Data and Radiological Characteristics}

There were 14 males and 11 females in the study group; their mean age was $30.90 \pm 13.52$ years (range 9-56 years). The comparison group had identical sex and age characteristics. The mean duration of symptoms was $34.08 \pm 42.40$ months (range 3-180 months). Twelve (48\%) patients complained of neck pain at presentation. None of the patients had scoliosis. The radiological findings of the cohort are listed in Table 1.

\section{Muscle Areas in Control Versus Study Groups}

Compared with the non-CMI comparison group, the mean PSM/bony area ratio for RCmi and all of the subaxial muscles was lower in the study group, whereas the RCma CSA/VBA ratio was higher (Fig. 3). Of these values, the mean DE CSA/VBA ratio was significantly lower in the study group $(\mathrm{p}=0.04)$. Figure 4 compares the muscle areas in a 35-year-old male patient with those of an age- and sex-matched control subject.

\section{Cervical Taper Ratios}

The cervical taper ratio $(\mathrm{C} 1-7)$ was $-0.39 \pm 0.24 \mathrm{~mm} /$ level for the comparison group (Fig. 5 right) and $-0.84 \pm$ $0.52 \mathrm{~mm} /$ level for the study group (Fig. 5 left). The difference between these values was significant $(\mathrm{p}=0.0003)$.

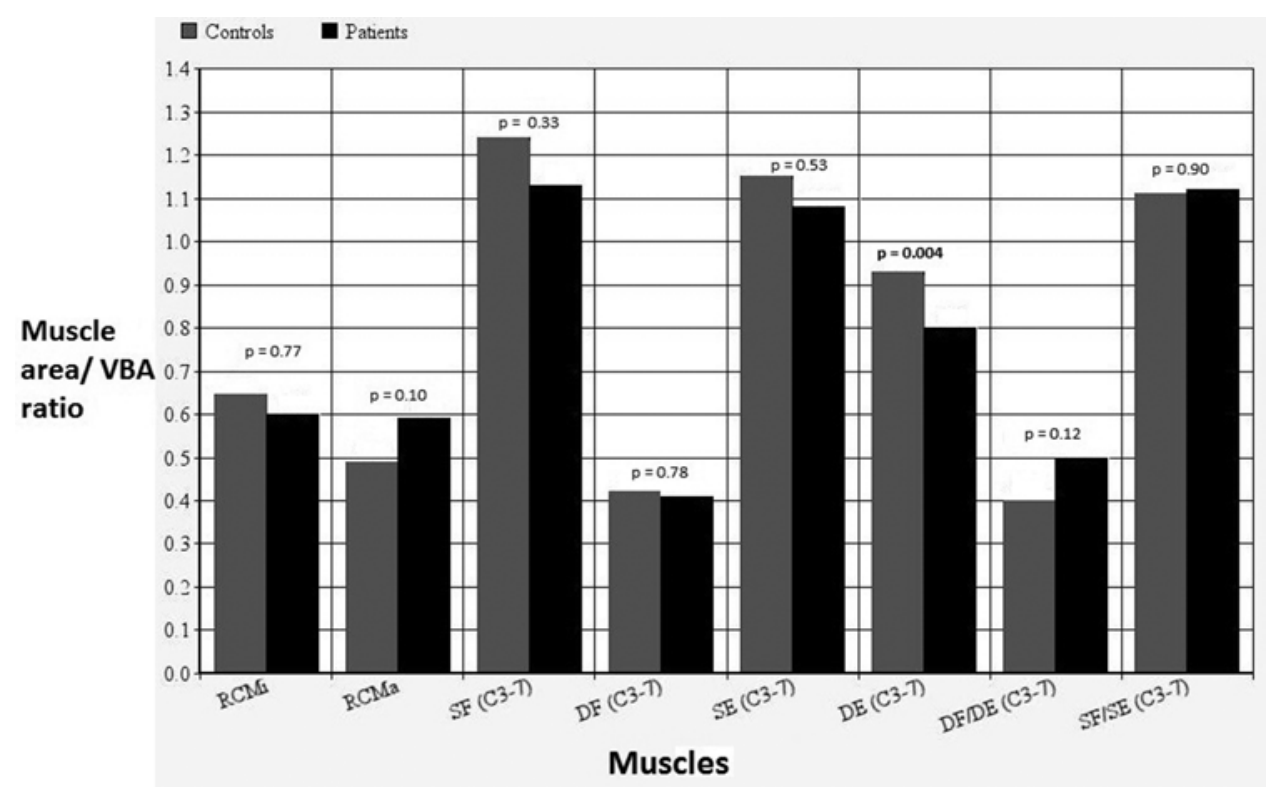

FIG. 3. Bar graph comparing the mean PSM area/bony area ratios in the control subjects and patients. 

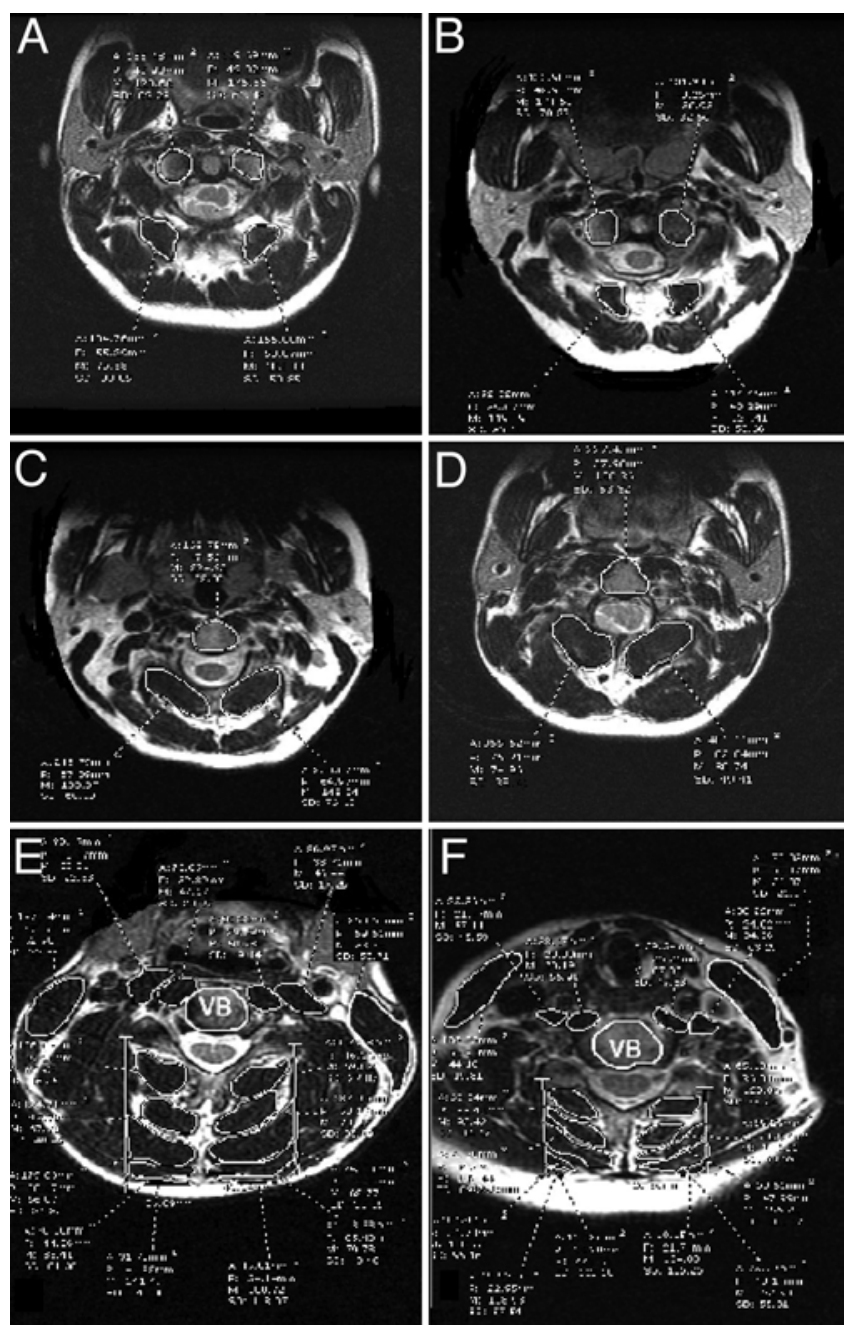

FIG. 4. Illustrative example comparing PSM areas in a 35-year-old man with those in an age- and sex-matched control. T2-weighted axial MRI sections demonstrate a larger $\mathrm{RCmi}$ area in the control subject $(\mathbf{A})$ than in the patient (B), a smaller RCma area in the control subject (C) than in the patient (D), and larger areas of the subaxial muscles in the control subject $(\mathbf{E})$ than in the patient $(\mathbf{F})$.

\section{Muscle Areas in Relation to Neck Pain Status}

The mean muscle areas of CMI subgroups with and without neck pain were compared with those of the nonCMI comparison group (Table 2). The subgroup of patients with CMI with neck pain demonstrated a smaller mean DF CSA/VBA ratio compared with that of the non-CMI group $(p=0.05)$. Compared with the non-CMI group, the
DE CSA/VBA ratio was significantly smaller in both of the CMI subgroups $(\mathrm{p}<0.05)$.

\section{Bivariate Analysis}

Pearson's correlation analysis (Table 3) demonstrated a strongly negative correlation of duration of symptoms with the DE CSA/VBA ratio $(r=-0.54)$. The cervical taper ratio demonstrated a strongly positive correlation with the SE CSA/VBA ratio $(r=0.65)$, a moderately positive correlation with the DF CSA/DE CSA ratio $(r=0.45)$, and a strongly negative correlation with the SF CSA/SE CSA ratio $(\mathrm{r}=-0.53)$. There was no significant correlation of sex, presence or absence of neck pain, and CSF flow on cine MRI with any of the muscle ratios $(\mathrm{p}>0.05)$.

\section{Multiple Regression Analysis}

A linear regression analysis was performed to evaluate the influence of age, sex, duration of symptoms, PFV/ICV, CSF flow on cine MRI, tonsillar herniation, taper ratio, and presence of neck pain on the PSM CSA/VBA ratios (Table 4). Assessing the overall fit of the linear regression model, the variables were found to significantly predict the subaxial muscle CSA/VBA ratios. Of the variables tested, female sex was independently associated with lower muscle CSA/VBA ratios for the SF, DF, and SE PSMs. A longer duration of symptoms and a steeper taper ratio were significantly associated with a lower DE CSA/VBA ratio, and the presence of neck pain was associated with a lower DF CSA/VBA ratio.

\section{Interobserver Variability}

The agreement between the observers for measurement of the PSM areas was substantial (weighted kappa coefficients: 0.71 for the suboccipital muscles, 0.78 for the subaxial muscles), and the agreement for the measurement of taper ratio and PFV/ICV was almost perfect (weighted kappa coefficients: 0.97 and 0.94 , respectively).

\section{Discussion}

The natural history and pathophysiology of CMI remain unclear. Various clinicoradiological phases in its pathophysiological continuum include a presyrinx state, ${ }^{6}$ syringomyelia, ${ }^{6,19}$ and scoliosis. ${ }^{19}$ The deleterious effect of CMI on cervical PSMs becomes obvious in the setting of scoliosis, the pathological end point of CMI. PSM atrophy at this stage is attributed to the effect of preceding syringomyelia on the anterior/posterior horn cells. The occurrence of scoliosis in the absence of preceding syringomy-
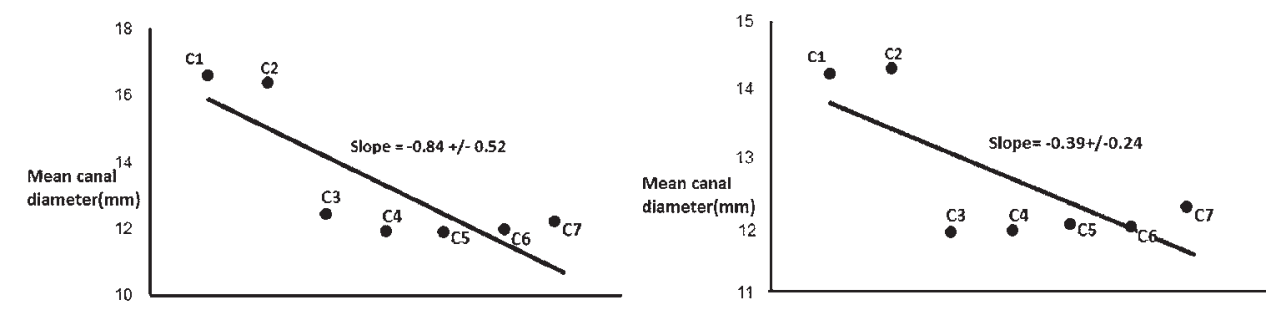

FIG. 5. Cervical canal taper ratio slope from C-1 to C-7 in patients with CMI (left) and in control subjects (right). 
TABLE 2. Muscle areas in comparison group versus patients with and without neck pain

\begin{tabular}{|c|c|c|c|c|c|}
\hline Muscle & Comparison Group $(n=25)^{*}$ & Patients w/o Neck Pain $(n=13)^{*}$ & p Value & Patients w/ Neck Pain $(n=12)^{*}$ & $p$ Value \\
\hline RCmi CSA/OCA & $0.65 \pm 0.44$ & $0.56 \pm 0.22$ & 0.49 & $0.60 \pm 0.32$ & 0.72 \\
\hline RCma CSA/VBA & $0.49 \pm 0.16$ & $0.58 \pm 0.20$ & 0.13 & $0.60 \pm 0.19$ & 0.07 \\
\hline SF CSA/VBA & $1.24 \pm 0.50$ & $1.16 \pm 0.33$ & 0.60 & $1.08 \pm 0.16$ & 0.28 \\
\hline DF CSA/VBA & $0.42 \pm 0.12$ & $0.45 \pm 0.17$ & 0.53 & $0.34 \pm 0.10$ & 0.05 \\
\hline SE CSA/VBA & $1.15 \pm 0.40$ & $1.13 \pm 0.47$ & 0.89 & $1.02 \pm 0.30$ & 0.32 \\
\hline DE CSA/VBA & $0.93 \pm 0.11$ & $0.81 \pm 0.18$ & 0.01 & $0.79 \pm 0.14$ & 0.002 \\
\hline DF CSA/DE CSA & $0.40 \pm 0.12$ & $0.50 \pm 0.21$ & 0.06 & $0.41 \pm 0.21$ & 0.85 \\
\hline SF CSA/SE CSA & $1.11 \pm 0.27$ & $1.10 \pm 0.32$ & 0.91 & $1.05 \pm 0.27$ & 0.53 \\
\hline
\end{tabular}

* Values are presented as the mean \pm SD.

$\dagger p$ values are for t-test comparisons of the patient subgroups with the comparison group.

elia in CMI has been explained by asymmetrical tonsillar herniation and dorsal column compression ${ }^{22}$ contributing to a loss of spinal alignment and ensuing PSM weakness. Some studies have established reversibility of the PSM changes $^{23}$ and degree of scoliosis ${ }^{18}$ following surgical intervention in CMI. Given these facts, it is surprising that in a developmental disorder with so many PSM-related implications, there has been no study that has analyzed their morphometric alterations.

\section{Alterations in PSMs in Other Conditions}

PSM area and function have been studied in normal subjects and in various disorders. Age ${ }^{15}$ bone mineral density, ${ }^{13}$ body mass index,${ }^{3}$ hormonal influence, ${ }^{13}$ and physical training ${ }^{17}$ are some of the factors that have been demonstrated to alter PSM CSA or function. Conditions such as whiplash-associated disorders (WAD), cervicogenic headache, ${ }^{10,12}$ and cervical spondylotic myelopathy ${ }^{21}$ reportedly cause a dysfunction of the deep cervical flexors. Dysfunction of the deep cervical extensors has been documented in some studies on patients with WADs..$^{1,2}$ Fatty infiltration in the suboccipital muscles has been noted in chronic neck pain, ${ }^{14}$ and selective dysfunction of the RCmi muscle has been implicated in chronic tension-type headache. ${ }^{5}$ The deep PSMs and the suboccipital muscles are predominantly composed of Type I fibers, with a high density of muscle spindles. Alteration of afferent input from these fibers explains the disturbances in proprioceptive function and kinesthetic sense in these disorders. ${ }^{14}$

\section{Atrophy of PSMs in CMI}

Compared with the normative control subjects, patients with CMI in our study demonstrated atrophy of the RCmi and all of the subaxial muscles. Generalized involvement of the cervical PSMs in a pathology at the CVJ could be attributed to neck pain-related disuse or fatty infiltration, dorsal column compression by the tonsils at the CVJ (alluded to earlier), or dysfunction of the anterior/posterior horn cells by altered CSF dynamics within the spinal cord. Although age did not affect the PSM areas, smaller areas of the flexors and SEs were more common in the female sex. Neck pain in the CMI subjects in our study correlated well with DF atrophy, a finding that is similar to that in other studies on chronic neck pain.

\section{Implication of Cervical Myodural Bridges in CMI}

Cervical myodural bridges are anatomical soft tissue bridges across the cervical epidural space that connect the suboccipital muscles to the dura mater.,16 Postulated to play a role in monitoring dural tension, these bridges prevent dural buckling and maintain patency of the spinal canal. ${ }^{4}$ Alterations in their actions are of relevance in the setting of a crowded subarachnoid space in CMI. The RCma areas were found to be higher in our study cohort compared with the control subjects. This may be attributed to a compensatory hypertrophy in the setting of dysfunctional cervical myodural bridges. Our finding lends credence to the proposition that failure of the myodural bridges and

TABLE 3. Correlations between muscle area/bony area ratios and various factors

\begin{tabular}{lccccc}
\hline \multirow{2}{*}{ Muscle } & Age & Duration of Symptoms & PFV/ICV & Tonsillar Herniation & Taper Ratio \\
\cline { 2 - 6 } & $0.17(0.46)$ & $-0.09(0.70)$ & $-0.06(0.78)$ & $0.12(0.60)$ & $0.01(0.17)$ \\
\hline RCmi CSA/OCA & $0.25(0.25)$ & $-0.06(0.79)$ & $-0.21(0.33)$ & $0.09(0.70)$ & $0.09(0.68)$ \\
\hline RCma CSA/VBA & $0.10(0.63)$ & $-0.27(0.21)$ & $-0.29(0.19)$ & $0.18(0.43)$ & $0.29(0.18)$ \\
\hline SF CSA/VBA & $0.32(0.14)$ & $-0.32(0.14)$ & $-0.33(0.13)$ & $0.21(0.35)$ & $0.26(0.22)$ \\
\hline DF CSA/VBA & $0.27(0.21)$ & $-0.36(0.09)$ & $-0.24(0.27)$ & $0.39(0.07)$ & $0.65(0.001)$ \\
\hline SE CSA/VBA & $0.18(0.43)$ & $-0.54(0.01)$ & $-0.24(0.28)$ & $0.31(0.16)$ & $0.18(0.41)$ \\
\hline DE CSA/VBA & $0.36(0.09)$ & $-0.04(0.85)$ & $-0.13(0.55)$ & $0.10(0.65)$ & $0.45(0.03)$ \\
\hline DF CSA/DE area & $0.22(0.30)$ & $0.41(0.05)$ & $-0.01(0.96)$ & $0.34(0.12)$ & $-0.53(0.009)$ \\
\hline SF CSA/SE area & $0.22)$ &
\end{tabular}


TABLE 4. Linear regression analysis

\begin{tabular}{|c|c|c|c|c|c|c|c|c|c|c|c|c|c|c|c|c|c|c|}
\hline \multirow[b]{2}{*}{ Variable } & \multicolumn{3}{|c|}{ SF CSA/VBA } & \multicolumn{3}{|c|}{ DF CSA/VBA } & \multicolumn{3}{|c|}{ SE CSA/VBA } & \multicolumn{3}{|c|}{ DE CSA/VBA } & \multicolumn{3}{|c|}{ DF/DE } & \multicolumn{3}{|c|}{ SF/SE } \\
\hline & B & $\begin{array}{l}\text { St } \\
E\end{array}$ & $\begin{array}{c}p \\
\text { Value }\end{array}$ & B & $\begin{array}{l}\text { St } \\
\mathrm{E}\end{array}$ & $\begin{array}{c}p \\
\text { Value }\end{array}$ & B & $\begin{array}{l}\text { St } \\
\mathrm{E}\end{array}$ & $\begin{array}{c}p \\
\text { Value }\end{array}$ & B & $\begin{array}{l}\text { St } \\
\mathrm{E}\end{array}$ & $\begin{array}{c}p \\
\text { Value }\end{array}$ & B & $\begin{array}{l}\text { St } \\
E\end{array}$ & $\begin{array}{c}\mathrm{p} \\
\text { Value }\end{array}$ & B & $\begin{array}{l}\text { St } \\
\mathrm{E}\end{array}$ & $\begin{array}{c}p \\
\text { Value }\end{array}$ \\
\hline Age & 0.02 & 0.01 & 0.97 & 0.78 & 0.00 & 0.09 & 0.08 & 0.01 & 0.79 & 0.46 & 0.01 & 0.32 & 0.12 & 0.01 & 0.82 & -0.18 & 0.01 & 0.69 \\
\hline Sex & 0.66 & 0.16 & 0.04 & -0.63 & 0.07 & 0.03 & -0.42 & 0.14 & 0.03 & -0.06 & 0.11 & 0.82 & -0.57 & 0.13 & 0.09 & -0.05 & 0.15 & 0.86 \\
\hline Duration & -0.01 & 0.00 & 0.97 & -0.15 & 0.00 & 0.51 & -0.31 & 0.00 & 0.05 & -0.30 & 0.00 & 0.04 & 0.15 & 0.00 & 0.58 & 0.65 & 0.00 & 0.05 \\
\hline PFV/ICV & -0.17 & 5.35 & 0.68 & -0.72 & 2.44 & 0.06 & 0.12 & 4.59 & 0.62 & -0.67 & 3.47 & 0.99 & -0.04 & 4.13 & 0.91 & -0.33 & 5.00 & 0.37 \\
\hline Cine $(A)$ & 0.15 & 0.21 & 0.69 & -0.46 & 0.10 & 0.19 & 0.28 & 0.18 & 0.24 & -0.25 & 0.14 & 0.48 & -0.14 & 0.16 & 0.72 & -0.16 & 0.20 & 0.65 \\
\hline Cine $(P)$ & 0.14 & 0.28 & 0.73 & 0.66 & 0.13 & 0.08 & -0.14 & 0.24 & 0.57 & 0.80 & 0.18 & 0.05 & -0.02 & 0.22 & 0.95 & 0.24 & 0.26 & 0.51 \\
\hline $\begin{array}{l}\text { Tonsillar } \\
\text { herniation }\end{array}$ & -0.15 & 0.01 & 0.62 & -0.26 & 0.00 & 0.33 & 0.03 & 0.01 & 0.86 & 0.41 & 0.01 & 0.16 & -0.58 & 0.01 & 0.85 & -0.27 & 0.01 & 0.32 \\
\hline Taper ratio & 0.03 & 0.21 & 0.94 & -0.53 & 0.10 & 0.15 & 0.52 & 0.18 & 0.05 & -0.92 & 0.14 & 0.03 & 0.43 & 0.16 & 0.32 & -0.47 & 0.20 & 0.21 \\
\hline Neck pain & -0.55 & 0.23 & 0.21 & -0.89 & 0.10 & 0.03 & -0.27 & 0.19 & 0.28 & -0.46 & 0.15 & 0.25 & -0.39 & 0.18 & 0.38 & -0.43 & 0.15 & 0.28 \\
\hline
\end{tabular}

$A=$ anterior; $B=$ regression coefficient; $P=$ posterior; $S t E=$ standard error.

Various subaxial muscle area/VBA ratios are the constants; various clinical and radiological factors are the variables.

the allied structures could result in altered CSF flow; ${ }^{4}$ this deserves further research.

\section{Taper Ratio and Its Correlations With PSMs in CMI}

It has been documented that the cervical spinal canal tapers mildly from rostral to caudal in healthy subjects. ${ }^{20}$ A few studies have found this taper ratio to be greater in $\mathrm{CMI}^{7,8}$ and more so in patients with nondistended syringes $^{24}$ or scoliosis.7 The steeper tapering in CMI possibly results in higher CSF pressure gradients between the cranial and caudal ends of the spinal canal, resulting in dysfunctional CSF flow that favors the formation of a syrinx.$^{7,8}$ It has been suggested that this bony variation in the cervical spine could be a component of the mesodermal derangement of CMI. ${ }^{24}$ Our study has again demonstrated significantly steeper cervical taper ratios in patients with CMI compared with control subjects. Although bivariate analysis demonstrated that the taper ratio was correlated with other PSM areas, multiple regression analysis established that it was independently associated with atrophy only in the DEs, the muscles that were found to be the most affected.

\section{Implications of the Study}

Among all of the PSM changes seen in our study cohort, the alteration in the DEs was statistically significant. It remains to be seen whether changes in the remaining cervical PSMs in CMI gain significance in studies with larger sample sizes. The findings of particular interest in our study are the strong correlations of a longer duration of symptoms and steeper cervical taper ratios with DE atrophy. Given that WADs and alterations in cervical extensor muscles are closely associated, it is possible that a steeper cervical taper ratio and the accompanying abnormal cranial-caudal CSF gradients in patients with CMI predispose their spinal cords to a whiplash-like condition. Because a history of preceding trauma was not apparent in our patients, it is plausible that the whiplash-like condition was engendered by repeated and unnoticed microtrauma in their anomalous cervical spines. The morphometric altera- tion in the DEs was demonstrable even without coexistent syringomyelia and became more pronounced over time.

In light of the above postulations, cervical mesodermal derangements in CMI may have a clinically significant predictive and prognostic value. Further research is warranted to confirm if PSM changes are demonstrable even in asymptomatic patients with CMI. A steep cervical taper ratio and/or PSM changes could then be additional indications for surgery in CMI, especially in the early part of its natural history when the role of surgery is controversial.

\section{Limitations of the Study}

The addition of a validated outcome measure for neck pain would have helped evaluate correlations of PSM areas with the degree of neck pain in CMI. Another limitation of our study is the unavailability of follow-up PSM CSA values, which would have provided information on their reversibility after surgery and correlations with postoperative clinical outcomes.

\section{Conclusions}

Patients with CMI demonstrate alterations in their cervical paraspinal musculature, even in the absence of coexistent syringomyelia. Their DE muscles undergo significant atrophic changes that worsen with the duration of their symptoms. This could be related to a significantly steeper cervical taper ratio that their cervical cords are exposed to. Neck pain in these patients is related to atrophy of their DF muscles. A steeper cervical taper ratio and alterations in the PSMs could be additional indicators for surgery in patients with CMI without syringomyelia.

\section{References}

1. Elliott J, Jull G, Noteboom JT, Darnell R, Galloway G, Gibbon WW: Fatty infiltration in the cervical extensor muscles in persistent whiplash-associated disorders: a magnetic resonance imaging analysis. Spine (Phila Pa 1976) 31:E847E855, 2006

2. Elliott J, Jull G, Noteboom JT, Galloway G: MRI study of 
the cross-sectional area for the cervical extensor musculature in patients with persistent whiplash associated disorders (WAD). Man Ther 13:258-265, 2008

3. Elliott JM, Jull GA, Noteboom JT, Durbridge GL, Gibbon WW: Magnetic resonance imaging study of cross-sectional area of the cervical extensor musculature in an asymptomatic cohort. Clin Anat 20:35-40, 2007

4. Enix DE, Scali F, Pontell ME: The cervical myodural bridge, a review of literature and clinical implications. J Can Chiropr Assoc 58:184-192, 2014

5. Fernández-de-Las-Peñas C, Cuadrado ML, Arendt-Nielsen L, Ge HY, Pareja JA: Association of cross-sectional area of the rectus capitis posterior minor muscle with active trigger points in chronic tension-type headache: a pilot study. Am J Phys Med Rehabil 87:197-203, 2008

6. Furtado SV, Reddy K, Hegde AS, Weinstein PR: Posterior fossa morphometry in symptomatic pediatric and adult Chiari I malformation. J Clin Neurosci 16:1449-1454, 2009

7. Hammersley J, Haughton V, Wang Y, del Rio AM: Tapering of the cervical spinal canal in patients with scoliosis with and without the Chiari I malformation. AJNR Am J Neuroradiol 33:1752-1755, 2012

8. Hirano M, Haughton V, Munoz del Rio A: Tapering of the cervical spinal canal in patients with Chiari I malformations. AJNR Am J Neuroradiol 33:1326-1330, 2012

9. Jull GA, O'Leary SP, Falla DL: Clinical assessment of the deep cervical flexor muscles: the craniocervical flexion test. J Manipulative Physiol Ther 31:525-533, 2008

10. Jull GA, Sterling M, Falla D, Treleaven J, O'Leary S (eds): Whiplash, Headache, and Neck Pain: Research-Based Directions for Physical Therapies. Edinburgh: Churchill Livingstone/Elsevier, 2008

11. Landis JR, Koch GG: The measurement of observer agreement for categorical data. Biometrics 33:159-174, 1977

12. Lee H, Nicholson LL, Adams RD: Neck muscle endurance, self-report, and range of motion data from subjects with treated and untreated neck pain. J Manipulative Physiol Ther 28:25-32, 2005

13. Leifke E, Körner HC, Link TM, Behre HM, Peters PE, Nieschlag E: Effects of testosterone replacement therapy on cortical and trabecular bone mineral density, vertebral body area and paraspinal muscle area in hypogonadal men. Eur J Endocrinol 138:51-58, 1998

14. McPartland JM, Brodeur RR, Hallgren RC: Chronic neck pain, standing balance, and suboccipital muscle atrophy-a pilot study. J Manipulative Physiol Ther 20:24-29, 1997

15. Okada E, Matsumoto M, Ichihara D, Chiba K, Toyama Y, Fujiwara $\mathrm{H}$, et al: Cross-sectional area of posterior extensor muscles of the cervical spine in asymptomatic subjects: a 10-year longitudinal magnetic resonance imaging study. Eur Spine J 20:1567-1573, 2011

16. Scali F, Pontell ME, Enix DE, Marshall E: Histological analysis of the rectus capitis posterior major's myodural bridge. Spine J 13:558-563, 2013
17. Sevki K, Mehmet T, Ufuk T, Azmi H, Mercan S, Erkal B: Results of surgical treatment for degenerative cervical myelopathy: anterior cervical corpectomy and stabilization. Spine (Phila Pa 1976) 29:2493-2500, 2004

18. Sha S, Zhu Z, Qiu Y, Sun X, Qian B, Liu Z, et al: [Natural history of scoliosis after posterior fossa decompression in patients with Chiari malformation/syringomyelia.] Zhonghua Yi Xue Za Zhi 94:22-26, 2014 (Chinese)

19. Strahle J, Smith BW, Martinez M, Bapuraj JR, Muraszko KM, Garton HJ, et al: The association between Chiari malformation Type I, spinal syrinx, and scoliosis. J Neurosurg Pediatr 15:607-611, 2015

20. Tatarek NE: Variation in the human cervical neural canal. Spine J 5:623-631, 2005

21. Thakar S, Mohan D, Furtado SV, Sai Kiran NA, Dadlani R, Aryan S, et al: Paraspinal muscle morphometry in cervical spondylotic myelopathy and its implications in clinicoradiological outcomes following central corpectomy: clinical article. J Neurosurg Spine 21:223-230, 2014

22. Tubbs RS, Doyle S, Conklin M, Oakes WJ: Scoliosis in a child with Chiari I malformation and the absence of syringomyelia: case report and a review of the literature. Childs Nerv Syst 22:1351-1354, 2006

23. Zhu Z, Sha S, Sun X, Liu Z, Yan H, Zhu W, et al: Denervation of the paraspinal muscles in patients with scoliosis secondary to Chiari malformation and syringomyelia: does it improve following posterior fossa decompression? Scoliosis 10 (1 Suppl):O11, 2015

24. Zhu Z, Sha S, Sun X, Liu Z, Yan H, Zhu W, et al: Tapering of the cervical spinal canal in patients with distended or nondistended syringes secondary to Chiari type I malformation. AJNR Am J Neuroradiol 35:2021-2026, 2014

\section{Disclosures}

The authors report no conflict of interest concerning the materials or methods used in this study or the findings specified in this paper.

\section{Author Contributions}

Conception and design: Thakar, Kurudi Siddappa. Acquisition of data: Thakar, Kurudi Siddappa. Analysis and interpretation of data: Thakar, Aryan, Mohan, Sai Kiran. Drafting the article: Thakar, Aryan, Mohan. Critically revising the article: Thakar, Kurudi Siddappa, Aryan. Reviewed submitted version of manuscript: all authors. Approved the final version of the manuscript on behalf of all authors: Thakar. Statistical analysis: Thakar. Study supervision: Hegde.

\section{Correspondence}

Sumit Thakar, Department of Neurological Sciences, Sri Sathya Sai Institute of Higher Medical Sciences, Bangalore 560066, India. email: sumit.thakar@gmail.com. 